\title{
Folbp1 promotes embryonic myocardial cell proliferation and apoptosis through the WNT signal transduction pathway
}

\author{
SHU-PING HAN ${ }^{*}$, YA PAN ${ }^{*}$, YU-ZHU PENG ${ }^{1}$, XIAO-QI GU ${ }^{1}$, RONG-HUA CHEN ${ }^{2}$ and XI-RONG GUO ${ }^{1,2}$ \\ ${ }^{1}$ Department of Pediatrics, Nanjing Maternity and Child Care Hospital Affiliated of Nanjing Medical University, \\ 210004 Nanjing; ${ }^{2}$ Department of Pediatrics, Nanjing Medical University, 210029 Nanjing, P.R. China
}

Received October 14, 2008; Accepted December 8, 2008

DOI: 10.3892/ijmm_00000134

\begin{abstract}
The aim of this study was to evaluate the relationship between Folate-binding protein one (Folbp1) and embryonic cardiac proliferation, apoptosis, and differentiation. Folbp1 gene expression and short interference RNA expression vectors were constructed. Morphology of P19 cells during differentiation was observed by inverted microscope. Cell proliferation was tested using the 3-(4,5-Dimethylthiazol-2-yl)2,5-Diphenyltetrazoliumbromide (MTT) method. Cell apoptosis was evaluated by DNA ladder and flow cytometry methods, and marker gene expression during differentiation, such as atrial natriuretic peptide (ANP) and cardiac troponin I (cTnI), and marker gene expression during apoptosis (Bax/Bcl-2) was measured by RT-PCR. Additionally, the critical genes (Wnt, GSK3ß, and/or ß-catenin) expressed in the Wnt signaling pathway were confirmed by RT-PCR. The Folbp1 expressing vector and the silencing vector were constructed. From day 5 of differentiation, the absorbance of cells overexpressing Folbp1 was notably higher than the controls, whereas the controls were notably higher than Folbp1 gene silenced P19 cells. P19 cell apoptosis with Folbp1 gene silencing was lower than the controls; however, more cells were driven into $\mathrm{S}$ phase. No significant morphological difference was observed in any of the groups. RTPCR results show that ANP, cTnI, Wnt, Bax/Bcl-2, and Bcatenin were elevated whereas GSK3B depressed in cells which overexpressed Folbp1 and was contradictory in Folbp1 gene silenced P19 cells. Folbp1 may be an important candidate mediator of folic acid deficiency-induced congenital
\end{abstract}

Correspondence to: Dr Xi-rong Guo, Department of Pediatrics, Nanjing Maternity and Child Care Hospital Affiliated of Nanjing Medical University, 210004 Nanjing, P.R. China

E-mail: xrguo@njmu.edu.cn

Dr Rong-hua Chen, Department of Pediatrics, Nanjing Medical University, 210029 Nanjing, P.R. China

E-mail: rhchen@njmu.edu.cn

${ }^{*}$ Contributed equally

Key words: folate deficiency, heart development, Folate-binding protein one, Wnt signal transduction pathway, offspring cardiac anomalies which are induced by the dysfunction of proliferation and apoptosis of the myocardial cell, and possibly caused by the dysfunction of the Wnt signaling pathway.

\section{Introduction}

In higher vertebrates, heart formation is a complex process that begins at early stages of embryogenesis with commitment of anterior lateral plate mesoderm cells to the cardiogenic lineage. These committed precursors subsequently differentiate into cardiomyocytes, and the heart develops principally through proliferation of these differentiated myocytes (1). A delicate balance between cell differentiation, proliferation, and apoptosis is crucial for proper organogenesis. Cell-specific transcription factors, which play pivotal roles in cell fate determination, are critical to an understanding of the molecular pathways underlying cell behavior during organ formation. Folate is known to be essential in many cellular reactions, including synthesis of nucleotides and cell cycle regulation (2). Periconceptional folate supplementation is widely believed to aid in the prevention of neural tube defects (NTDs), orofacial clefts, and congenital heart defects (CHDs) (3). Folate deficiency during pregnancy in humans and mice has been shown to affect heart development, leading to CHDs and myocardial hypoplasia. Maternal folate supplementation is believed to prevent human CHDs $(3,4)$.

The beneficial effect of additional folic acid in the periconceptional period to prevent conotruncal heart defects in offspring has been shown. Folate-binding protein one (Folbp1) is the primary mediator of folic acid transport into murine cells. Folbp1 functions as a membrane receptor mediating high-affinity internalization and delivery of folate to cell cytoplasm. In vitro, this receptor facilitates the accumulation of cellular folate a thousand-fold relative to the media; therefore, it is essential in cytoplasmic folate delivery in vivo (5). Folbp1 knockout mouse embryos die in utero with multiple malformations, including severe CHDs (6). Administration of folate to Folbp1-deficient dams before and throughout gestation rescues the majority of embryos from premature death (7-9). Although maternal folate supplementation is believed to prevent human CHDs, its precise role during cardiac morphogenesis remains unclear. We built Folbp1 overexpressioning and RNA interference silencing expression vectors and explored the effects of folate on myocardial cell proliferation, apoptosis, and differentiation. We also examined 
the Wnt signal transduction pathways importance in embryonic cardiac development.

Wnt proteins (Wnts) are secreted glycoproteins that are highly insoluble. Wnts function as growth factors and morphogens which modulate cell growth and specify cell fate, thereby playing a key role in the developmental process in embryogenesis, including generation of cell polarity, specification of cell fate, and regulation of proliferation and differentiation (10). Additionally, Wnt-dependent signals can involve either a canonical B-catenin pathway or other alternative mediators. At the cell surface, Wnt proteins activate the pathway through binding a receptor complex consisting of Frizzled, intracellular mediators of the canonical pathway include $\beta$-catenin, which promotes pathway activation, and GSK-3ß, which negatively regulates the pathway. In the absence of Wnt ligand, cytoplasmic levels of B-catenin protein remain low as a result of its association with GSK-3ß, which targets $\beta$-catenin protein for proteolytic degradation. Upon Wnt ligand binding, GSK-3ß is inhibited and cytoplasmic levels of $\beta$-catenin protein increase and subsequently translocate into the nucleus, where $\beta$-catenin functions as a transcriptional co-activator (11). Previous research has shown that Wnt is possibly one of the important factors that could induce or enhance cardiac differentiation by regulating the silent gene expressions; however, the relationship of gene expression in the WNT signal transduction pathway with the Folbp1 gene and embryonic cardiac development remains unclear. It is known that P19 cells are pluripotent mouse cell lines when exposed to DMSO, and differentiate to embryonic myocardial cells; they are useful model systems for investigating the roles of various factors in cardiac induction and differentiation (12). Therefore, we evaluated the relationship of Folbp1 with embryonic cardiac proliferation, apoptosis, and differentiation, as well as the effect of Folbp1 on gene expression regulation in the Wnt signaling pathway of P19 cells.

\section{Materials and methods}

P19 cell culture and induction of differentiation. The embryonal carcinoma cells (P19 cells) were cultivated as aggregates from days 0 to 4 in the presence of $1 \% \alpha$-MEM containing $10 \%$ FBS in bacteriological dishes in $5 \% \mathrm{CO}_{2}$ at $37^{\circ} \mathrm{C}$. At day 4 , the cell aggregates were transferred to cell culture flasks. Cells were harvested at differentiation day 10 . Differentiation was induced when the medium was changed to $\alpha$-MEM containing $10 \mathrm{ml} / 1 \mathrm{Me}_{2} \mathrm{SO}$ (DMSO). After $48 \mathrm{~h}$, the medium was replaced with $\alpha$-MEM containing $10 \%$ FBS, and then was changed with the same medium every 2 days thereafter. Morphological changes of P19 cells were examined under a Nikon inverted microscope (Nikon, Japan) equipped with phase-contrast objectives and a digital camera (Nikon).

Establishment of Folbp1-expressing transformants. The coding sequence of Folbp1 was subcloned into the HindIII and $\mathrm{XbaI}$ sites of a pcDNA 3.1/myc-His B expression vector using oligonucleotides 5'-CCCAAGCTTGCCACCATGC TCACCTGATGACT-3' and 5'-GCTCTAGAGGCTGATCA CCCAGAGCAGCACT-3' to generate a plasmid expressing Folbp1-6X His fusion protein. Expression vectors carrying the Folbp1 coding sequence or empty vectors were transfected into P19 cells. Two days after transfection, neomycin (G418, Switzerland) was added to the medium $(400 \mu \mathrm{g} / \mathrm{ml})$ to select transfected cells. Drug-resistant cells began to form small colonies after two weeks of G418 addition. Individual colonies were isolated and propagated, and Folbp1-6X His fusion protein was identified by Western blotting using an anti-6X His antibody (Clontech, USA). Colonies expressing the highest levels of Folbp1 were selected for cell proliferation and differentiation studies. The resultant plasmids were confirmed by sequencing. The predicted molecular weight of the Folbp1 His fusion protein was $22.7 \mathrm{kDa}$ (not including the signal peptide).

Western blotting. Total proteins were isolated from cultured cells using the TRIzol method (Invitrogen, USA). Total proteins were separated on a $7.5 \%$ gel by SDS-PAGE and transferred to a polyvinylidene difluoride membrane. Membranes were incubated with mouse anti-6X His antibody (Clontech, USA) and goat anti-mouse immunoglobulin G (IgG)-horseradish peroxidase conjugate (Amersham, UK). Immunoreactive proteins were detected by enhanced chemiluminescence (Amersham).

Construction of Folbpl short interference RNA (siRNA) expression plasmids. BLAST homology searching was used to identify 3 conserved cDNA fragments within the coding region of mouse Folbp1 genes. From these sequences, we designed and synthesized 3 pairs of reverse complementary oligonucleotides, each containing a loop sequence (5'-TTC AAGAGA-3'), the RNA PloIII terminator (5'-TTTTTT-3'), and 5 ' single-stranded overhangs for ligation into HindIII- and BamHI-digested pcDNA 6.2-GW/EmGFP-miR (Invitrogen) vectors (Ambion, USA). These oligonucleotides were annealed and inserted into the siRNA expression vectors. The sequences of the 2 cDNA fragments (sense strands) were: (1) 5'-CCT TTCATTTCTCCTCCTT-3'; (2) 5'-AGCTGCTCCTGT GGCTCTG-3'. These were used to create pcDNA 6.2-GW -folbp1-1, and pcDNA 6.2-GW -folbp1-2, respectively. An insert control designed to target GAPDH mRNA was used as the positive control (Ambion). An insert-free pcDNA 6.2-GW vector served as the negative control.

RNA interference. Four pcDNA 6.2-GW/EmGFP-miR vectors including a negative and a positive control were Fugene 6transfected into untreated or Folbp1-transfected P19 cells, which were then grown in 6-well culture plates with complete culture medium. An LPS-free vector ( $2 \mathrm{mg}$ per well) was used for each transfection. The ratio of fugene 6 to the vector was 6:1.2 days (48 h) after transfection, cells and supernatants were collected for real-time PCR detection.

Real-time qRT-PCR. Mass aggregates were pooled, centrifuged, and resuspended in $0.5 \mathrm{ml}$ chilled guanidinium thiocyanate (GTC) buffer. Monolayer cultures were solubilized directly with $0.5 \mathrm{ml}$ buffer. Dissociated cells were centrifuged and resuspended in the buffer. In all cases, DNA was sheared by passing the extract 20 times through a 16-gauge needle. The samples were then flash-frozen in liquid nitrogen and stored at $80^{\circ} \mathrm{C}$ until use. Total RNA was extracted as described 
Table 1. Primers for RT-PCR amplification.

\begin{tabular}{|c|c|c|c|c|}
\hline Gene name & Product size (bp) & Reverse and forward primer & $\mathrm{Ta}\left({ }^{\circ} \mathrm{C}\right)$ & Cycles \\
\hline ANP & 148 & $\begin{array}{l}\text { R: 5'-TGGGCTCCAATCCTGTCAATC-3' } \\
\text { F: 5'-GAGACGGCAGTGCTCTAGG-3' }\end{array}$ & 60 & 27 \\
\hline CTnI & 117 & $\begin{array}{l}\text { R: 5'-GATCCTCACTCTTCGGAGGGT-3" } \\
\text { F: 5'-AGATATGACGTGGAAGCAAAAGT-3' }\end{array}$ & 60 & 33 \\
\hline Wnt & 212 & $\begin{array}{l}\text { R: 5'-CGTTTAGCAGTTTTGTCAGCTC-3' } \\
\text { F: 5'-CGAAGGCTCCATCGAGTCC-3' }\end{array}$ & 60 & 30 \\
\hline B-catenin & 189 & $\begin{array}{l}\text { R: 5'-CGTTTAGCAGTTTTGTCAGCTC-3' } \\
\text { F: 5'-TCCCTGAGACGCTAGATGAGG-3' }\end{array}$ & 60 & 25 \\
\hline GSK3ß & 125 & $\begin{array}{l}\text { R: 5'-ACCAGGTTAAGGTAGACCTCATC-3' } \\
\text { F: 5'-GACAAGCGATTTAAGAACCGAGA-3' }\end{array}$ & 60 & 30 \\
\hline Bax & 229 & $\begin{array}{l}\text { R: 5'-CCAGCCCATGATGGTTCTGAT-3' } \\
\text { F: 5'-CCGGCGAATTGGAGATGAACT-3' }\end{array}$ & 60 & 33 \\
\hline Bcl-2 & 284 & $\begin{array}{l}\text { R: 5'-CAGACATGCACCTACCCAGC-3' } \\
\text { F: 5'-GTCGCTACCGTCGTGACTTC-3' }\end{array}$ & 60 & 30 \\
\hline GAPDH & 251 & $\begin{array}{l}\text { R: 5'-GCCAGTAGAGGCAGGGATGATGTTC-3' } \\
\text { F: 5'-CCATGTTCGTCATGGGTGTGAACCA-3' }\end{array}$ & 60 & 28 \\
\hline
\end{tabular}

ANP, atrial natriuretic peptide; CTnI, Cardiac troponin I; R, reverse primer; F, forward primer; Ta, annealing temperature.

$\begin{array}{llll}1 & 2 & 3 & \text { Marker }\end{array}$

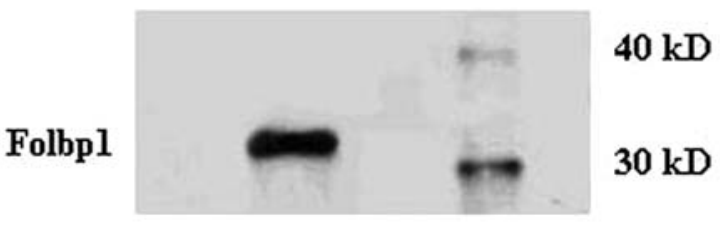

Figure 1. Expression of Folbp1 protein in P19 cells transfected with pcDNA3.1-Folbp1. Total proteins were isolated from stable cell lines and analyzed by Western blotting using a mouse anti-6X His antibody. Lane 1, normal P19 cell; Lane 2, pcDNA3.1-folbp1; lane 3, pcDNA3.1. Each experiment was repeated 3 times and results were coincident.

previously (13). Samples were treated with amplification grade RNase-free DNase I (Invitrogen). Total RNA (1.5 $\mu \mathrm{g})$ was reverse transcribed in a volume of $60 \mu \mathrm{l}$. As a negative control, reverse transcriptase was omitted in duplicate reverse transcription reactions. For real-time PCR, $2 \mu 1$ of cDNA (40 ng) was added to $18 \mu \mathrm{l}$ of Master mix containing 1U SYBR Green (Applied Biosystems, Foster City, CA) and $400 \mathrm{nM}$ sense and anti-sense primers. cDNA triplicates were amplified for 40 cycles either with the Applied Biosystems 7300 or with the Opticon I real-time thermal cycler (MJ Research, Inc., Waltham, MA). The PCR product level was calculated from the threshold cycle $(\mathrm{Ct})$, the amplification cycle at which the emission intensity of the product rises above a set threshold level. The mouse specific primers used in this study were: Folbp1 (fw): 5'-CGGAACTATGACATCGGAATGC-3' and (rv): 5'-CTCTTTGCACAGGGGAACATC-3'.

Cell growth analysis. To analyze the growth of stably transfected P19 cells, cells were seeded in 96-well culture plates at a density of $10^{3}$ cells/well and cultured in media supplemented with 10\% FBS. Untransfected P19 cells were also cultured as a control. Cell growth was analyzed by $3-$ (4,5-dimethylthiazol-2-yl)-2,5-diphenyltetrazoliumbromide (MTT) assays using the Cell proliferation MTT kit I (Roche, Switzerland) every $24 \mathrm{~h}$ for a period of 7 days. Absorbance was measured at $490 \mathrm{~nm}$.

Effects of Folbpl gene on the cell cycle of P19 cells. The effect of the Folbp1 gene on cell cycle was evaluated in Folbp1 gene silencing P19 cells or normal P19 cells. Before analysis, cells were serum deprived for $48 \mathrm{~h}$ to synchronize the cell cycle. Cells were incubated with $\alpha$-MEM containing $10 \%(\mathrm{v} / \mathrm{v})$ FBS for another $24 \mathrm{~h}$ after serum deprivation. Cell cycle distributions were assessed using methods described previously (14). Briefly, cells were fixed in $70 \%$ ethanol and cellular DNA was stained with propidium iodide. Total DNA content was analyzed in each cell line by flow cytometry.

Total RNA isolation and amplification by RT-PCR. Total RNA was isolated from cultured P19 cells using the TRIzol method (Invitrogen). Single strand cDNA synthesis was performed as follows: the reverse transcription mixture contained $1 \mu \mathrm{g}$ total RNA, $0.5 \mu \mathrm{g}$ of oligo d(T) primer, $4 \mu \mathrm{l}$ of $5 \mathrm{X}$ RT buffer, $0.5 \mathrm{mM}$ deoxynucleotides, $50 \mathrm{U}$ of RNase inhibitor, and $200 \mathrm{U}$ of reverse transcriptase (Promega, USA) in a total volume of $20 \mu \mathrm{l}$. The reaction was carried out at $42^{\circ} \mathrm{C}$ for $1 \mathrm{~h}$ followed by heat inactivation at $95^{\circ} \mathrm{C}$ for $5 \mathrm{~min}$. An aliquot of the cDNA was amplified with the primers listed in Table I. The number of cycles and reaction temperatures used in the PCR assay were optimized to provide a linear relationship between the amount of input template and the amount of PCR product. 
A

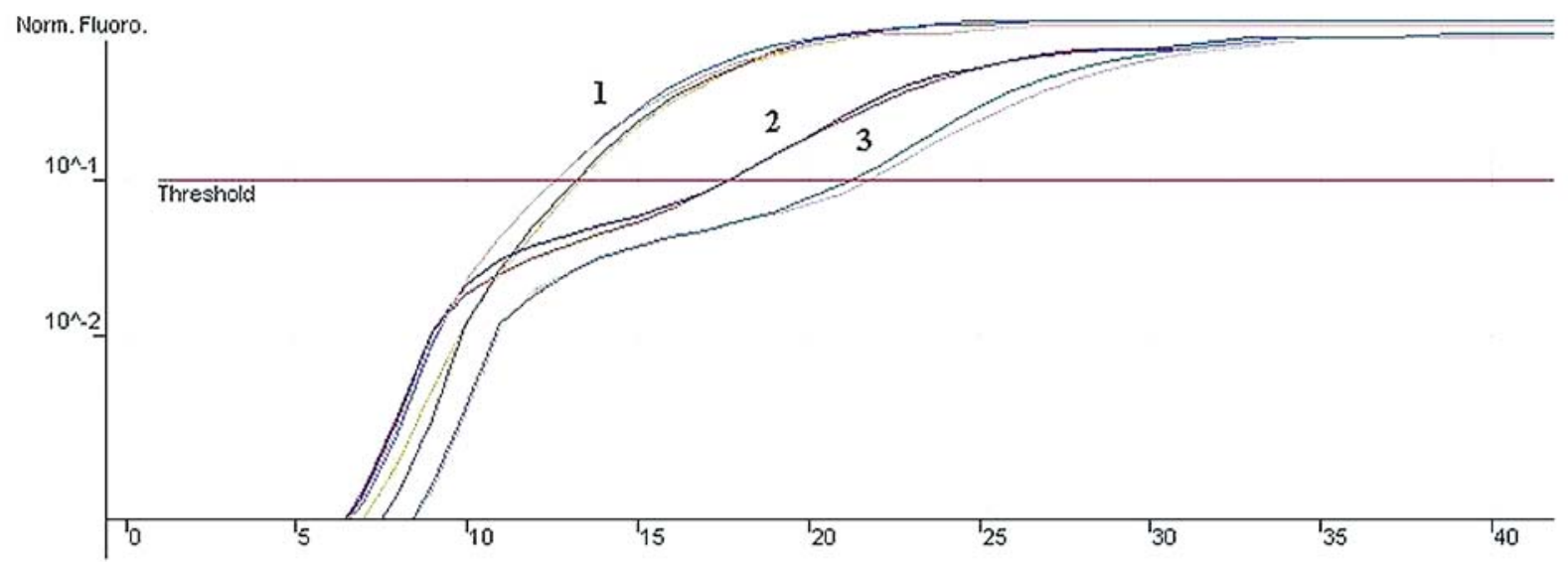

B
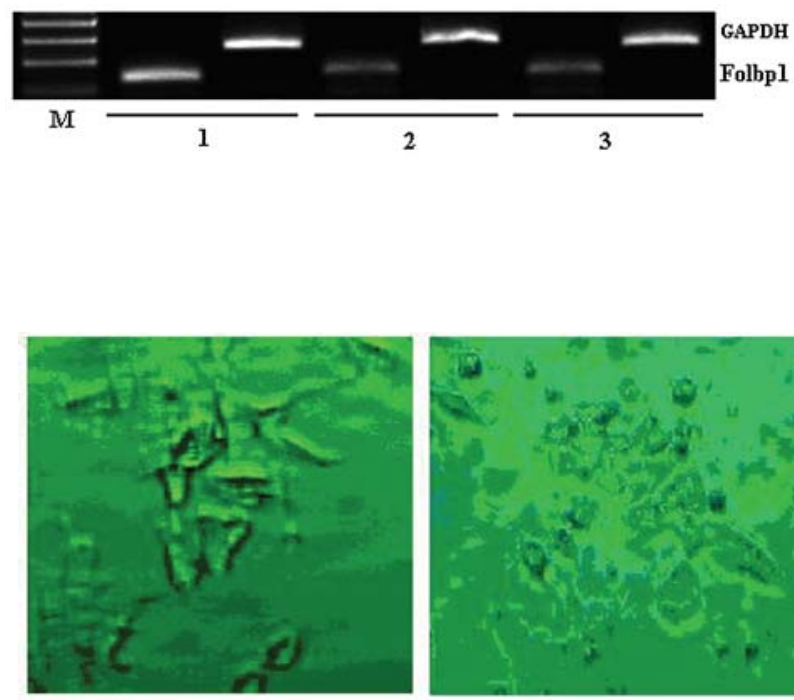

day 0 -control.

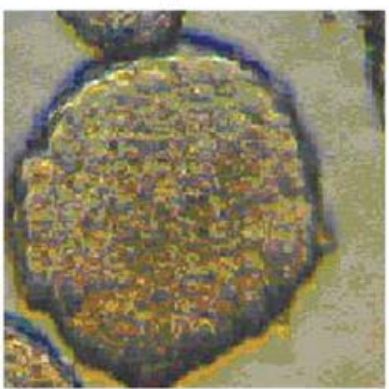

day 4-control

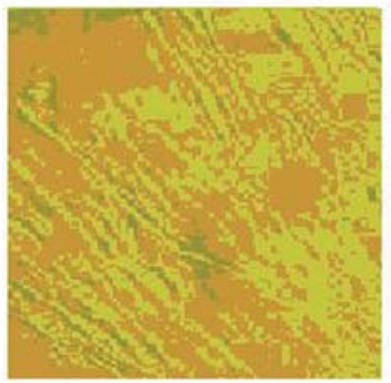

day 10 -control

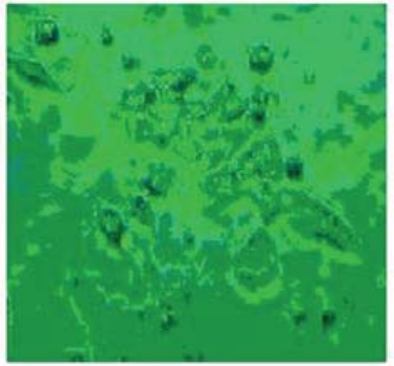

day 0 -overexoression

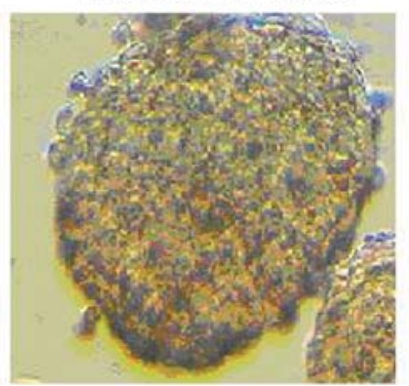

day 4-overexpression

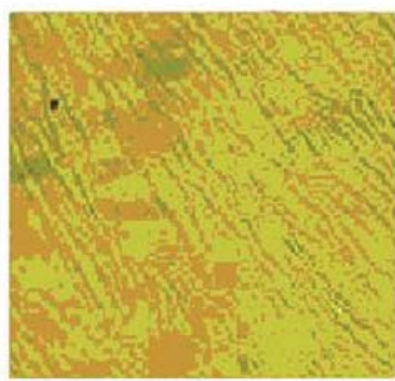

day 10-overexpression

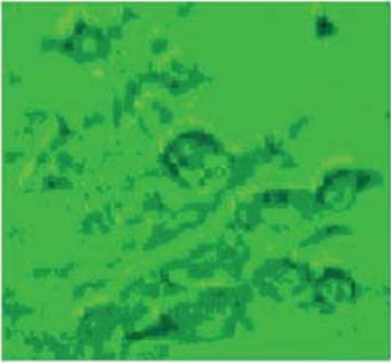

day $0-\mathrm{i}-1$

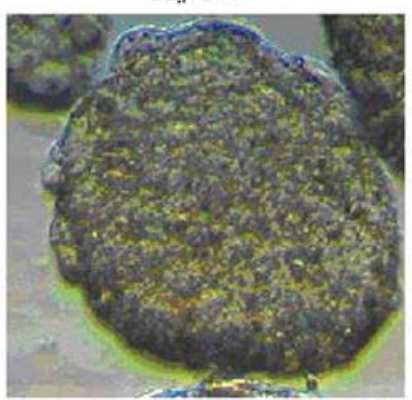

day4-i-1

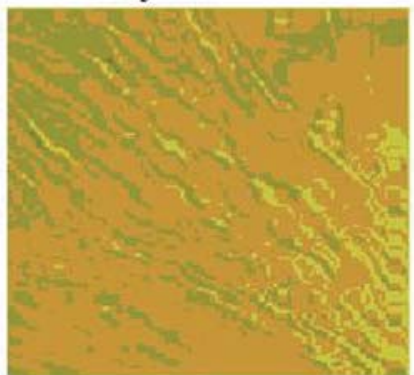

day $10-\mathrm{i}-1$

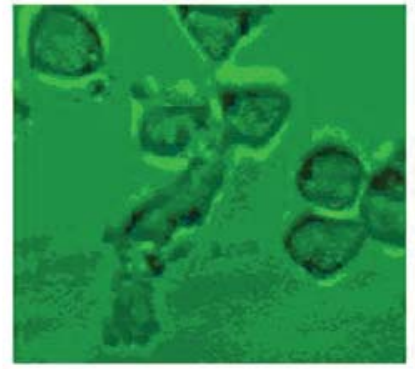

day $0-\mathrm{i}-2$

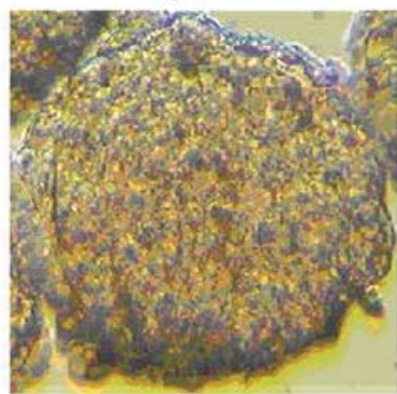

day4-i-2

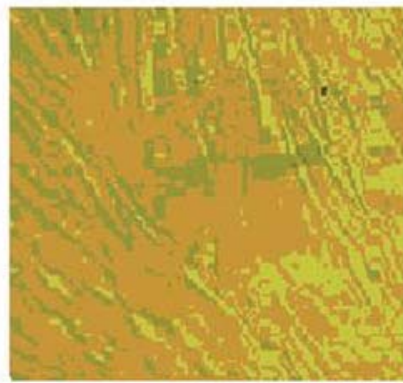

day $10-\mathrm{i}-2$

Figure 3. Morphology of P19 cells during differentiation. Control, P19 cells; overexpression, pcDNA 6.2-GW -Folbp1-1 P19 cells; P19-i-1, pcDNA 6.2GW -folbp1-1; P19-i-2, pcDNA 6.2-GW -folbp1-2. 
A
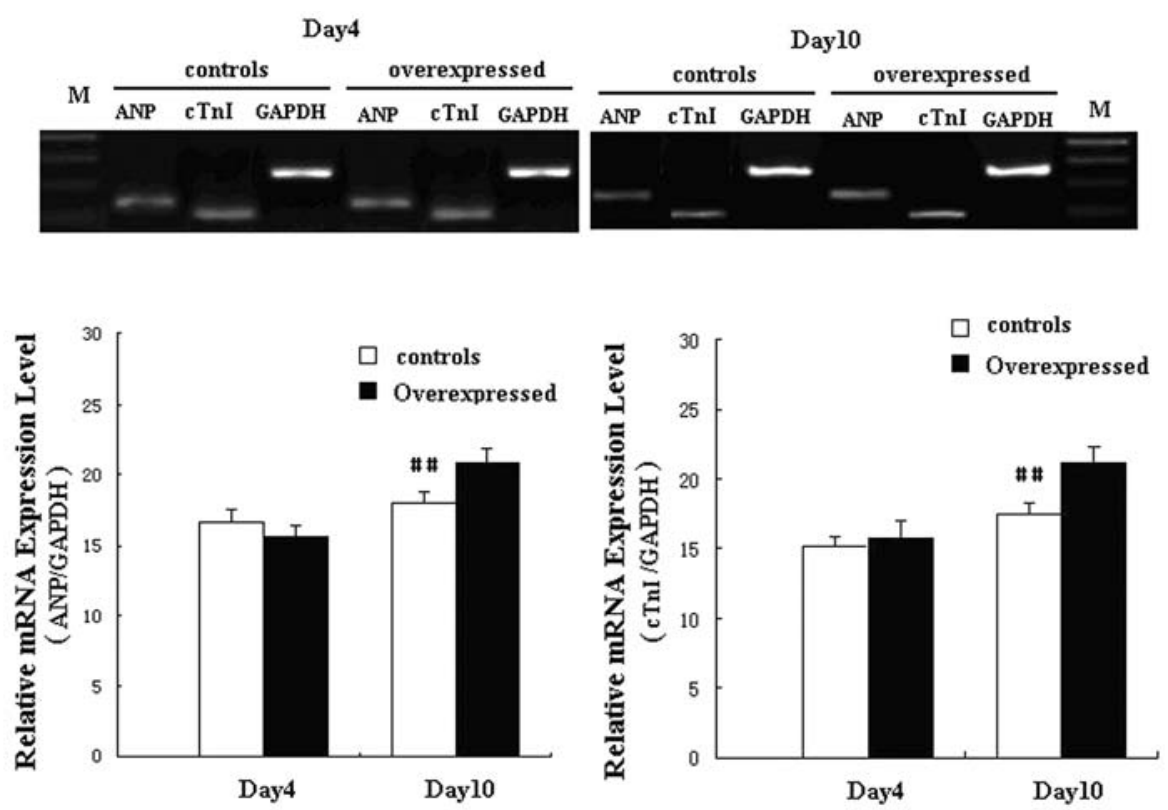

B

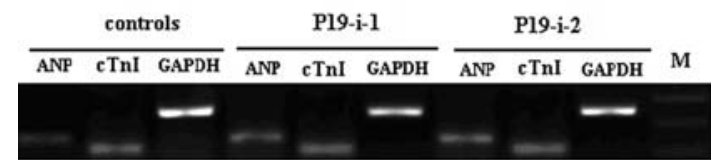

Day 4

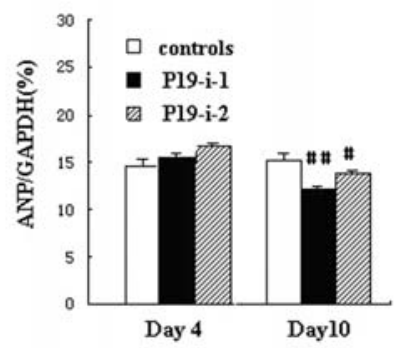

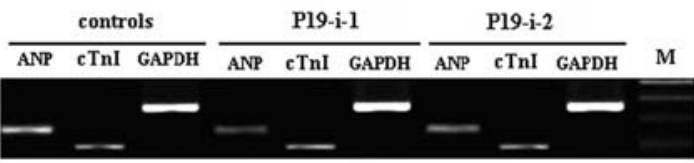

Day10

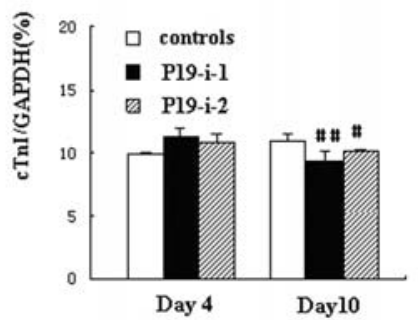

Figure 4. Expression of marker genes during P19 cell differentiation. (A) Folbp1 overexpression; (B) Folbp1 interferenced. All the cells were induced to differentiate and were collected at days 4 and 10. Total RNA was reverse transcribed, and an aliquot of cDNA was PCR amplified, electrophoresed on an ethidium bromide agarose gel, and visualized under ultraviolet light. Results are expressed as the ratio between the intensity of bands corresponding to the gene studied vs. the intensity of bands corresponding to GAPDH.

Statistical analysis. All results are expressed as mean \pm SD. Data were analyzed using one-way ANOVA and t- or t'-tests with a correction for multiple comparisons, as appropriate.

\section{Results}

Stable cell line overexpressing Folbp1. The P19 cell line stably transfected with a Folbp1 plasmid was established and maintained in $\alpha$-MEM containing $200 \mu \mathrm{g} / \mathrm{ml} \mathrm{G418.} \mathrm{Folbp1}$ protein expression was verified by Western blotting (Fig. 1).

siRNA inhibition of Folbpl expression. The P19 cell line stably transfected with a Folbp1-silencing plasmid was established and maintained in $\alpha$-MEM containing $200 \mu \mathrm{g} / \mathrm{ml} \mathrm{G} 418$. Realtime PCR was used to identify the Folbp1 gene (Fig. 2).

Morphology of P19 cells during differentiation. P19 cells were derived from the tumor of an early mouse embryo, they were pluripotent in nature, and differentiated into various cell types depending on the environment (15). Cellular aggregation is known to be a requirement for DMSO-induced differentiation of P19 cells into cardiac myocytes. It is known that efficient differentiation of P19 cells depends on the prior formation of non-adhering aggregates, and these cellular aggregates resemble the inner cell mass of an embryo, although the molecular events that occur during aggregation and that are necessary for cardiac differentiation are not fully understood (12). To investigate how P19 cells can be differentiated into cardiac cells, we examined morphological changes of the P19 cells. Morphology of P19 cell lines overexpressing or silencing gene Folbp1 on days 0, 4, and 10 of differentiation are shown in Fig. 3. The cardial myocardial cells aggregated on day 4 of differentiation, and the beating cell colonies generated on day 10 of differentiation. However, similar morphological changes and beating cell clusters were occasionally observed on the indicated differentiation days in 
A

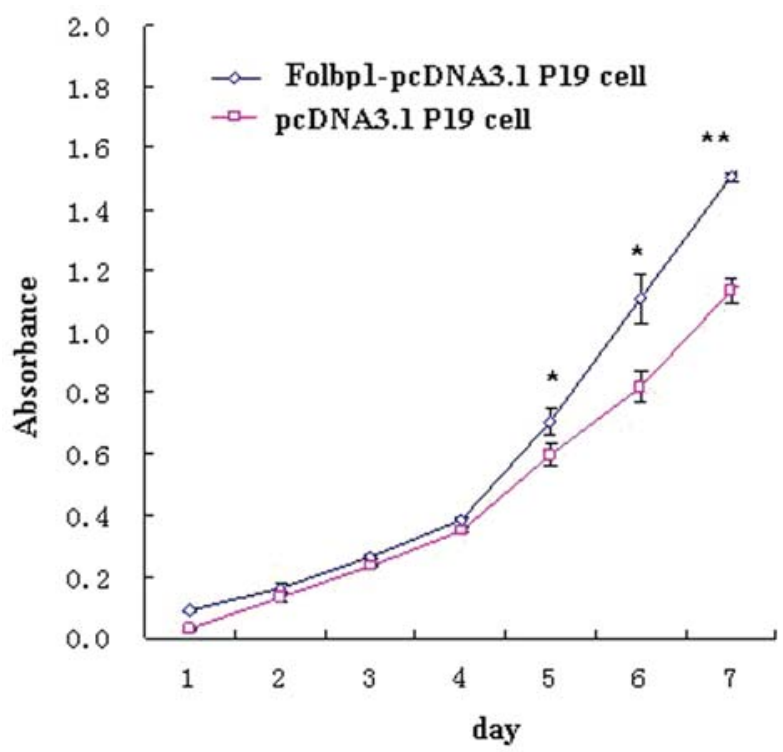

B

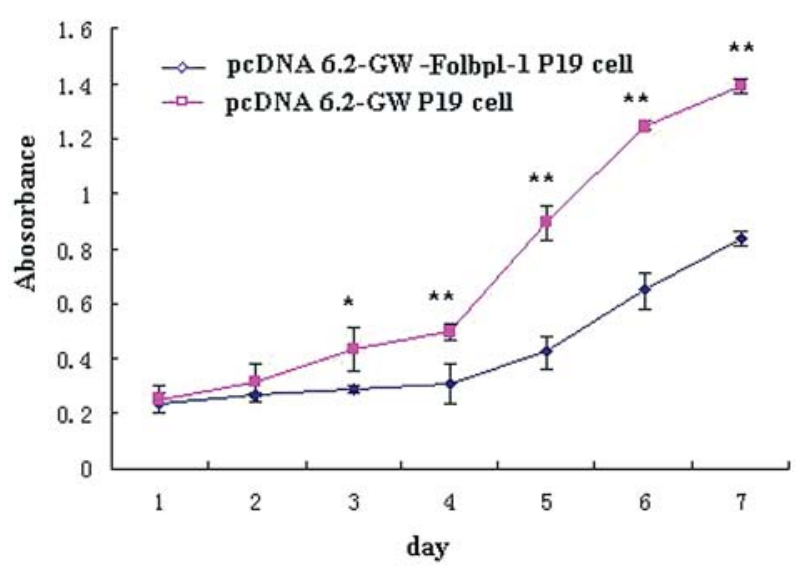

Figure 5. Growth curves during P19 cell proliferation and differentiation by MTT assay. (A) Folbp1 overexpressed; (B) Folbp1 interference. Data resulted from 3 independent experiments and are expressed as the mean \pm SEM of the difference between the absorbance at $490 \mathrm{~nm} .{ }^{*} \mathrm{p}<0.05$, ${ }^{* *} \mathrm{p}<0.001$.

the control cells, indicating that under these cultural conditions, the cells can spontaneously differentiate into myocardiocytes (Fig. 3).

Expression of marker genes during P19 cell differentiation. ES (embryonic stem) cell-derived cardiomyocytes express cardiac-specific genes and proteins in a developmentally controlled manner. For example, induction of cardiac troponin I (cTnI) mimics the isoform transition that occurs during in vivo cardiogenesis. Most atrial-specific protein genes, which include atrial natriuretic protein (ANP), are also expressed in ventricles during embryonic development. Therefore, ANP and cTnI expression was examined during P19 cell differentiation, as shown in Fig. 4. On day 10, ANP and $\mathrm{cTnI}$ expression was upregulated more than on day 4; this coincided with the character of the cardiacal myocardial differentiation. Though each cell line showed a consistent trend related to expression of these marker genes at day 4 , the ANP

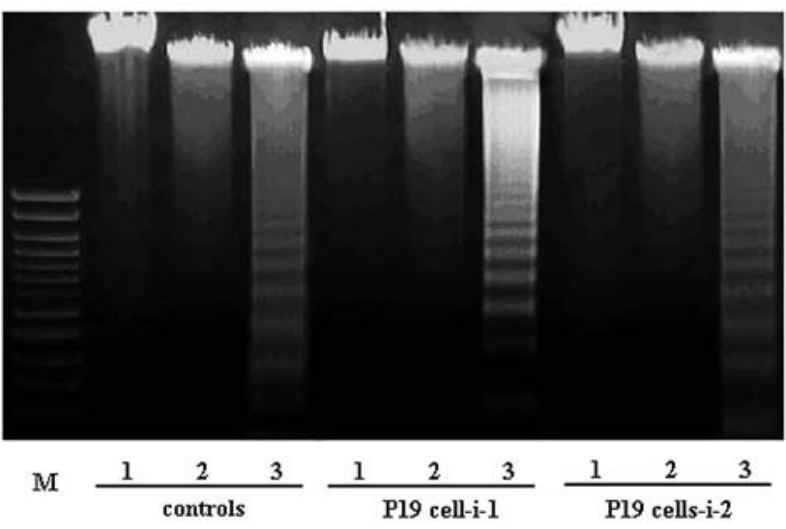

Figure 6. DNA ladder of P19 cells after RNA interference. 1, day 0; 2, day 4; 3, day 10. M, Marker; Controls, pcDNA 6.2-GW P19 cells; P19-i-1, pcDNA 6.2-GW -folbp1-1; P19-i-2, pcDNA 6.2-GW -folbp1-2.

A

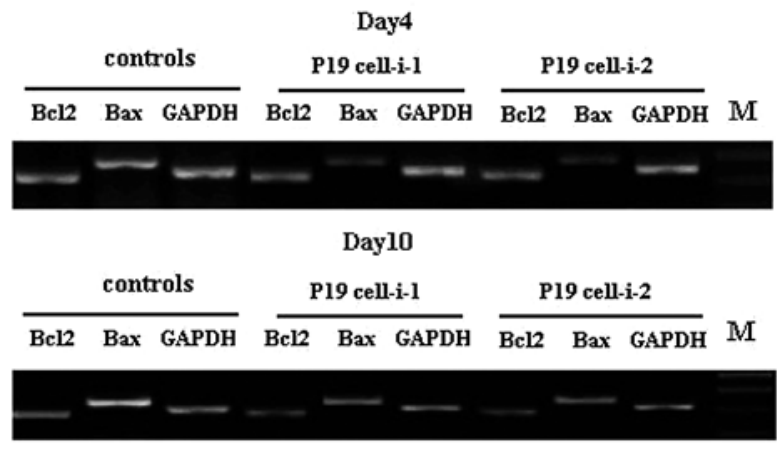

B

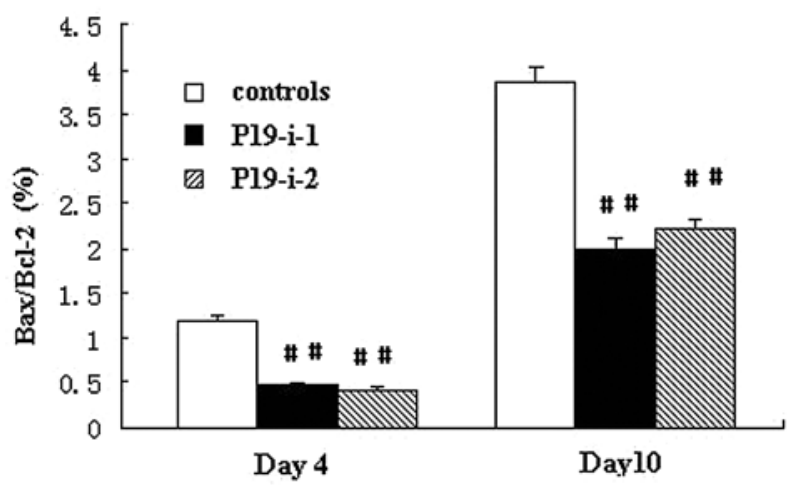

Figure 7. Expression of marker genes during P19 cell apoptosis by RT-PCR. (A) Differentiation of P19 cells at days 4 and 10. (B) Results are expressed as the ratio between the intensity of bands corresponding to the $\mathrm{Bax} / \mathrm{Bcl}-2$. Controls, pcDNA 6.2-GW P19 cells; P19-i-1, pcDNA 6.2-GW -folbp1-1; P19-i-2, pcDNA 6.2-GW -folbp1-2. Total RNA was reverse transcribed, and an aliquot of cDNA was PCR amplified, electrophoresed on an ethidium bromide agarose gel, and visualized under ultraviolet light. Each value represents the mean \pm SEM of 3 separate experiments. Statistical analysis was performed according to the Student's t-test, ${ }^{\# \#} \mathrm{p}<0.001$.

and cTnI expression in cells with overexpressed P19 cells was higher than the controls on day 10 of differentiation, while ANP and cTnI expression was lower as a result of interference (Fig. 4). The results show that Folbp1 can contribute the marker gene expression of myocardial cell differentiation 

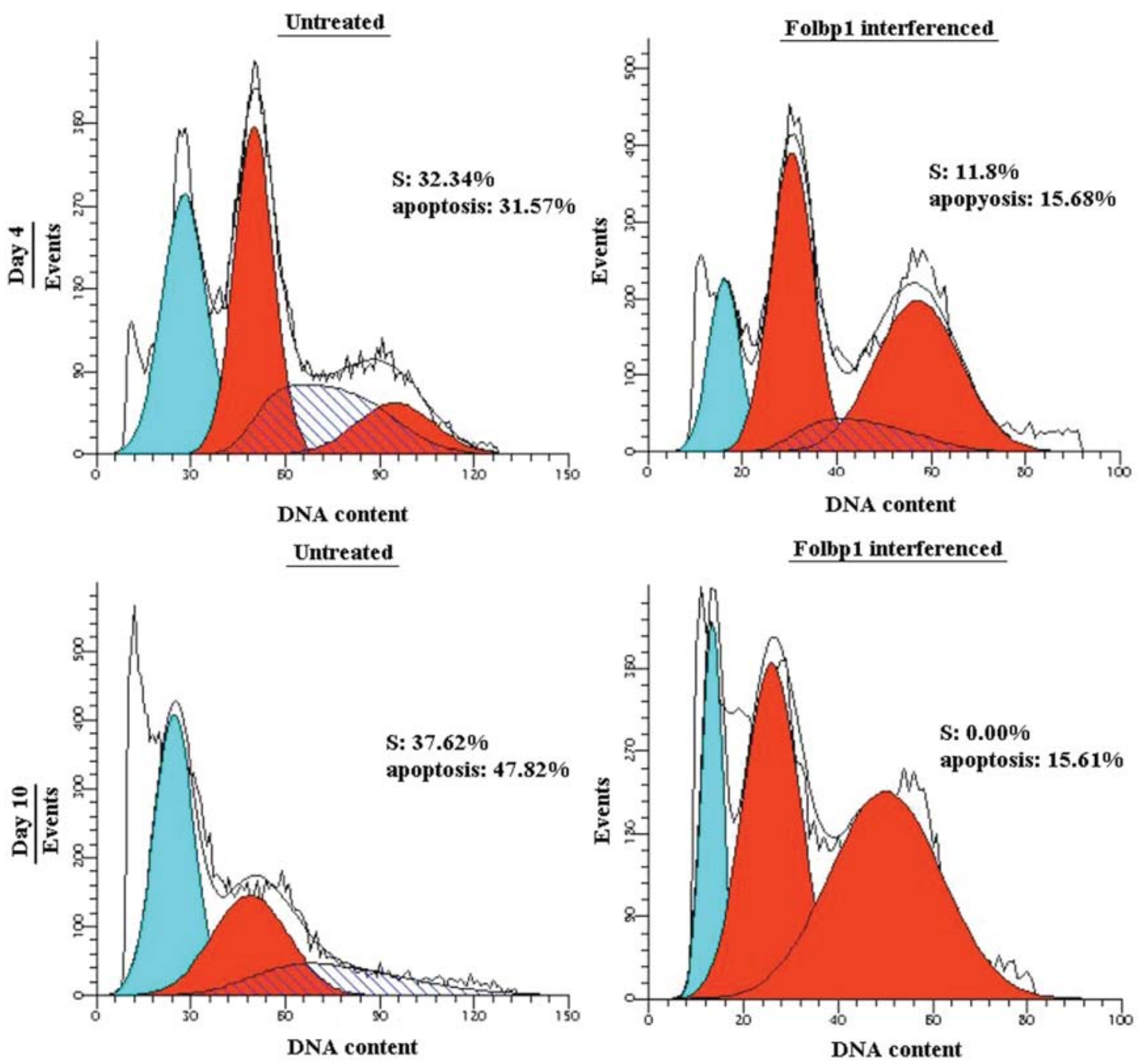

Figure 8. Cell cycle analysis of P19 cells transfected with Folbp1 gene silencing vector. P19 cells transfected with the folbp1 gene silencing vector and untransfected P19 cells serving as controls were cultured in serum-free media for $48 \mathrm{~h}$ and subsequently evaluated with Annexin-V assays, the results of total $\mathrm{S}$ phase and apoptosis are shown.

as, the changes in gene expression appeared earlier than the morphological changes.

Growth curves during P19 cell proliferation and differentiation. We generated growth curves by MTT assay to analyze the effect of Folbp1 on P19 cell growth (Fig. 5). The results show that from day 1 to 4 , the absorbance of cells expressing Folbp1 or the empty vector had not changed. From day 5, the absorbance of cells overexpressing Folbp1 was notably higher than the controls, while the P19 cells of silencing Folbp1 had a decreased growth rate compared with the controls. From day 1 to 2, the absorbance of silencing Folbp1 P19 cells or empty vectors had not changed. From day 3 , the absorbance of empty vector was notably higher than the Folbp1 gene silencing P19 cells. From the growth curve, we concluded that Folbp1 can contribute to P19 cell proliferation.

DNA ladder of P19 cells after RNA interference. To observe Folbp1 apoptosis of the P19 cells, genomic DNA fragmentation was tested. All P19 cells induced a ladder pattern (a hallmark of cell apoptosis). The results show that all cells underwent apoptosis at day 10 of differentiation (Fig. 6).
Expression of critical apoptosis genes during P19 cell differentiation. The regulatory signals of the apoptosis cascade are extremely complex; therefore, apoptotic cells were analyzed for gene expression of pro-apoptotic (Bax) and anti-apoptotic (Bcl-2) proteins at days 4 and 10 of differentiation by RT-PCR, as shown in Fig. 7. The ratio of Bax and Bcl-2 in the P19 cells following Folbp1 interference was much lower than that of the controls.

Cell cycle analysis of the P19 interference cells. The effect of Folbp1 on P19 cell growth was further examined by cell cycle analysis of stably-transfected cells. P19 cells transfected with the Folbp1 gene silencing vector and untransfected P19 cells serving as controls were cultured in serum-free media for $48 \mathrm{~h}$ and subsequently evaluated with Annexin-V assays. At day 4, the distribution of Folbp1 silencing cells after synchronization was $43.98 \%$ in G1 phase, $11.8 \%$ in S phase, and $44.22 \%$ in G2 phase. The distribution of normal P19 cells was $51.55 \%$ in G1 phase, $32.34 \%$ in S phase, and $16.11 \%$ in G2 phase (Fig. 8). At day 10, the distribution of cells in Folbp1 silencing cells after synchronization was $44.75 \%$ in G1 phase, $0 \%$ in $\mathrm{S}$ phase, and $55.25 \%$ in $\mathrm{G} 2$ phase. Distribution of normal P19 cells was $62.38 \%$ in G1 phase, $37.62 \%$ in S phase, and 
A

\begin{tabular}{|c|c|c|c|c|c|c|c|c|c|c|c|c|c|c|c|}
\hline \multicolumn{4}{|c|}{ Day } & \multicolumn{4}{|c|}{4 overexpressed } & \multicolumn{5}{|c|}{ Day 10} & \multicolumn{3}{|c|}{ overexpressed } \\
\hline 1 & 2 & 3 & 4 & 1 & 2 & 3 & 4 & 1 & 2 & 3 & 4 & 1 & 2 & 3 & 4 \\
\hline
\end{tabular}
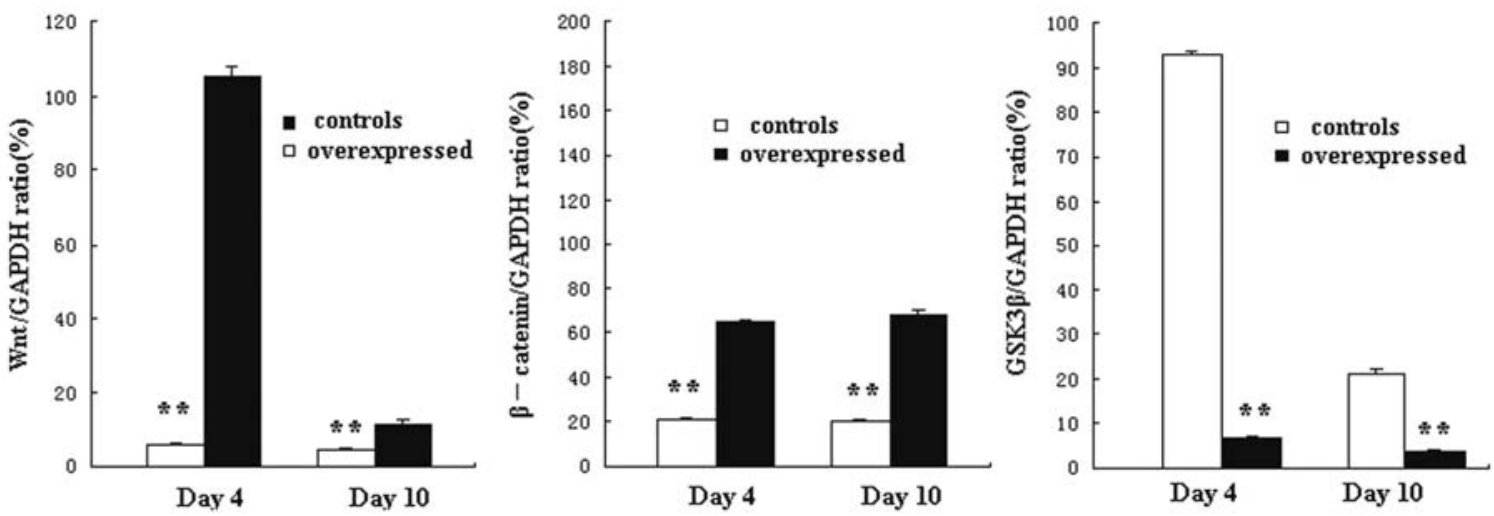

B

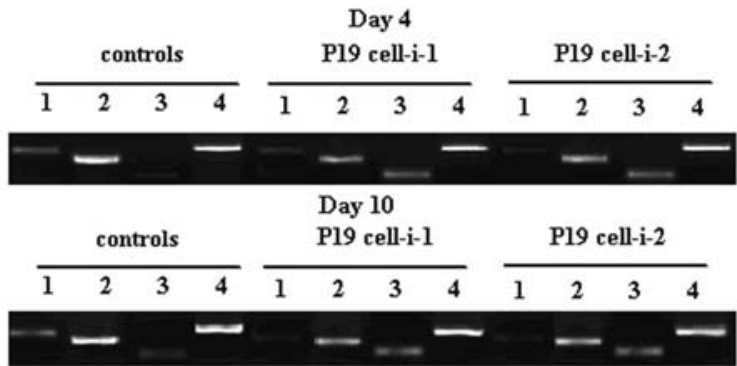
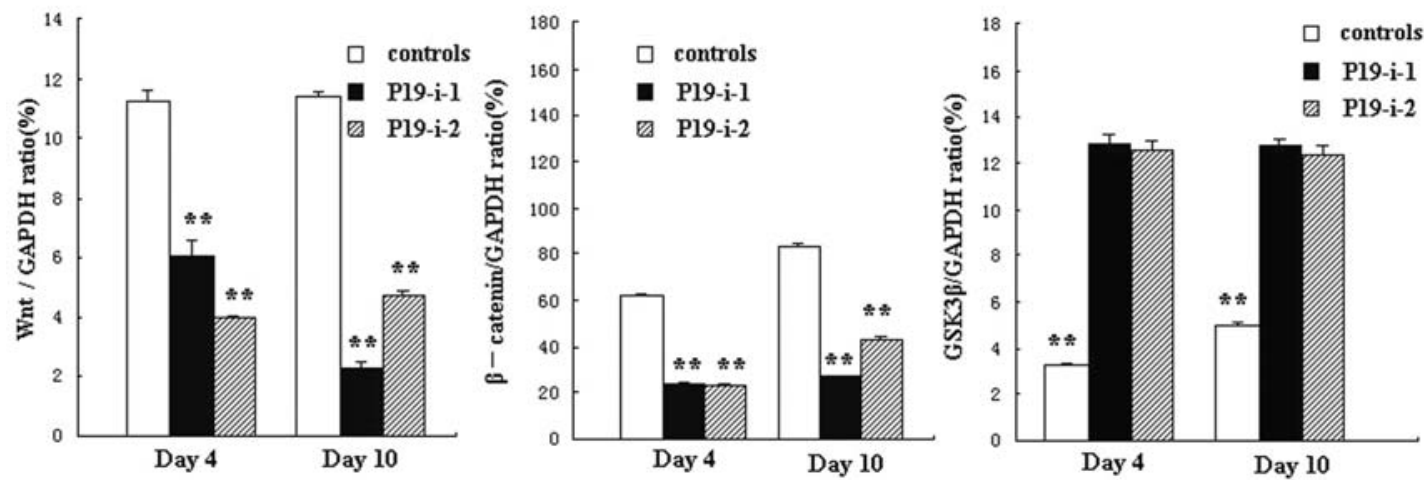

Figure 9. Expression of WNT signal transduction genes during P19 cell differentiation by RT-PCR. Results are expressed as the ratio between the intensity of bands corresponding to the gene studied vs. the intensity of bands corresponding to GAPDH. (A) Folbp1 expressing P19 cells. Overexpressed, Folbp1 expressing P19 cells; 1, Wnts; 2, B-catenin; 3, GSK3ß; 4, GAPDH. (B) Folbp1 silenced P19 cells. Controls, pcDNA 6.2-GW P19 cells; P19-i-1, pcDNA 6.2GW -folbp1-1; P19-i-2, pcDNA 6.2-GW -folbp1-2, 1, Wnts; 2, ß-catenin; 3, GSK3ß; 4, GAPDH.

$0 \%$ in G2 phase (Fig. 8). These results indicate that Folbp1 drives more P19 cells into $\mathrm{S}$ phase, which represents the cyto-chromatin synthesis period. The control cells grown in the absence of serum had a higher rate of apoptosis than the Folbp1 silenced cells (day 4, 31.57 vs. $15.68 \%$; day 10 , $47.82 \%$ vs. $15.61 \%)$.

Expression of WNT signal transduction genes during P19 cell differentiation. Folbp1 silenced cells, expressing P19 cells, and the controls were collected for up to 10 days. The expression levels of genes in the Wnt signal transduction pathway, such as Wnts, B-catenin, and GSK3ß, was measured by RT-PCR. Fig. 9 shows the expression levels of Wnts and ß-catenin were much higher in Folbp1 expressing P19 cells than in the controls, while they were much lower in Folbp1 gene silenced P19 cells. GSK3ß expression levels were significantly lower in Folbp1 expressing P19 cells than in the controls, while they were much higher in Folbp1 gene silenced P19 cells.

\section{Discussion}

Proliferation and apoptosis are presumed to play important roles during critical periods of heart development. Inadequate proliferation or excess apoptosis can directly or indirectly result in CHDs $(1,16)$, which often results from altered 
proliferation and/or apoptosis of the septum, neighboring tissue, or myocardium (17).

In the present study, we examined the potential influences of Folbp1 on myocardial cell proliferation and apoptosis. Results suggest that Folbp1 can promote P19 cell proliferation and apoptosis, thereby driving more P19 cells into S phase. Simultaneously, Folbp1 promotes marker gene expression during apoptosis. This indicates that Folbp1 is an important factor in cardiac proliferation and apoptosis, and is capable of altering related gene expression. These results are consistent with earlier studies which suggest that maternal folate status is associated with the risk of CHD. Li and Rozen (18) examined the processes in the myocardium of embryos from $\mathrm{Mthfr}^{+/+}$and $\mathrm{Mthfr}^{+/-}$mice fed control diets (CD) or folic acid-deficient diets (FADD) and found that the thickness of cardiac ventricular compact walls of embryos from FADDtreated dams was significantly reduced, and embryonic myocardium from FADD-treated dams had significantly fewer 5'-bromo-2'-deoxyuridine-labeled cells compared with CD-treated dams. These results suggest that proliferation of embryonic myocardium is sensitive to maternal dietary folate and that folate supplementation during pregnancy is important for normal heart development and prevention of heart defects.

There are several possible mechanisms by which folate can affect proliferation. Folate derivatives are involved in nucleotide synthesis, amino acid synthesis, and methylation reactions. During embryonic development, myocardial cells undergo rapid mitosis or differentiation. Thus, it is not surprising that folate deficiency can affect proliferation. Another metabolic consequence of folate deficiency is an elevation of homocysteine, which has been shown to inhibit proliferation in vitro $(19,20)$, and a decrease in methionine, which is required for the synthesis of S-adenosylmethionine for methylation reactions. Folate deficiency could therefore affect the expression of genes involved in cell proliferation through an effect on DNA methylation. The effect of folate deficiency on proliferation can account in part for the observed phenotypes (ventricular septal defects and myocardial hypoplasia) which are well-documented (21).

It is important to note that the results discussed above suggest that no difference in apoptosis was due to diet; however, our results suggest that the interference of Folbp1 in myocardial cells will downregulate apoptosis and related gene Bax/Bcl-2 expression. This may be because Li and Rozen (18) assessed apoptosis on gestational day 12.5 of the mouse pregnancy, when apoptosis of myocardial cells is not very obvious.

In the present study, we also investigated the effects of Folbp1 on P19 cell differentiation and found that P19 cells differentiate to myocardial cells regardless of the gene expression of Folbp1 almost simultaneously, while the marker gene during myocardial cell differentiation was elevated when Folbp1 was overexpressed.

In conclusion, we found that Folbp 1 can promote embryonic myocardial cell proliferation and apoptosis, but not differentiation. Folbp1 may be an important candidate mediator of folic acid deficiency-induced congenital cardiac anomalies which are induced by dysfunction of myocardial cell proliferation and apoptosis.
How specific areas of nondifferentiated mesoderm organize into myocardial tissue is a question that has long intrigued developmental biologists. In recent years, the regulation of Wnt signal transduction has been implicated as an important event that initiates cardiac development. Wnt signal transduction has received the most attention, as primary stimuli of cardiogenesis regulators. The Wnt gene family encodes for a conserved class of secreted signaling molecules and is considered one of the major gene families essential for proper embryonic patterning and organogenesis (22). In the present study, Wnt and B-catenin expression levels were elevated. Further, GSK3ß was depressed in the Folbp1 gene overexpressing P19 cells, while it was contradictory in the Folbp1 gene silenced P19 cells. Therefore, we conclude that congenital cardiac anomalies induced by the dysfunction of myocardial cell proliferation and apoptosis, which is induced by folate deficiency, may be caused by dysfunction of the WNT signaling pathway. Clarification of myocardial cell regulatory mechanisms under a folic acid-deficient state will provide further insight into the pathogenesis of heart development abnormalities in folic acid-deficient pregnancies.

\section{Acknowledgements}

Project supported by grants from the National Natural Science Foundation of China (No. 30672245) and the Medical Science and Technology Development Focal Point Foundation of Nanjing City (No. ZKX05011).

\section{References}

1. Gittenberger-de Groot AC, Bartelings MM, Deruiter MC and Poelmann RE: Basics of cardiac development for the understanding of congenital heart malformations. Pediatr Res 57: 169-176, 2005.

2. Scott JM: Reduced folate status is common and increases disease risk. It can be corrected by daily ingestion of supplements or fortification. Novartis Found Symp 282: 105-117, 2007.

3. McDonald SD, Ferguson S, Tam L, Lougheed J and Walker MC: The prevention of congenital anomalies with periconceptional folic acid supplementation. J Obstet Gynaecol Can 25: 115-121, 2003.

4. Botto LD, Mulinare J and Erickson JD: Do multivitamin or folic acid supplements reduce the risk for congenital heart defects? Evidence and gaps. Am J Med Genet 121: A95-A101, 2003.

5. Piedrahita JA, Oetama B, Bennett GD, et al: Mice lacking the folic acid-binding protein Folbp1 are defective in early embryonic development. Nat Genet 23: 228-232, 1999.

6. Tang LS, Santillano DR, Wlodarczyk BJ, Miranda RC and Finnell RH: Role of Folbp1 in the regional regulation of apoptosis and cell proliferation in the developing neural tube and craniofacies. Am J Med Genet C Semin Med Genet 135: C48-C58, 2005.

7. da Costa M, Sequeira JM, Rothenberg SP and Weedon J: Antibodies to folate receptors impair embryogenesis and fetal development in the rat. Birth Defects Res A Clin Mol Teratol 67: 837-847, 2003.

8. Zhu H, Wlodarczyk BJ, Scott M, et al: Cardiovascular abnormalities in Folr1 knockout mice and folate rescue. Birth Defects Res A Clin Mol Teratol 79: 257-268, 2007.

9. Spiegelstein O, Mitchell LE, Merriweather MY, et al: Embryonic development of folate binding protein-1 (Folbp1) knockout mice: Effects of the chemical form, dose, and timing of maternal folate supplementation. Dev Dyn 231: 221-231, 2004.

10. Nakamura T, Sano M, Songyang Z and Schneider MD: A Wntand beta-catenin-dependent pathway for mammalian cardiac myogenesis. Proc Natl Acad Sci USA 100: 5834-5839, 2003. 
11. Moon RT, Bowerman B, Boutros M and Perrimon N: The promise and perils of Wnt signaling through beta-catenin. Science 296: 1644-1646, 2002.

12. van der Heyden MA and Defize LH: Twenty one years of P19 cells: what an embryonal carcinoma cell line taught us about cardiomyocyte differentiation. Cardiovasc Res 58: 292-302, 2003.

13. Chomczynski P and Sacchi N: The single-step method of RNA isolation by acid guanidinium thiocyanate-phenol-chloroform extraction: twenty-something years on. Nat Protoc 1: 581-585, 2006.

14. Olivera A, Kohama T, Edsall L, et al: Sphingosine kinase expression increases intracellular sphingosine-1-phosphate and promotes cell growth and survival. J Cell Biol 147: 545-558, 1999.

15. McBurney MW, Jones-Villeneuve EM, Edwards MK and Anderson PJ: Control of muscle and neuronal differentiation in a cultured embryonal carcinoma cell line. Nature 299: 165-167, 1982.

16. Fisher SA, Langille BL and Srivastava D: Apoptosis during cardiovascular development. Circ Res 87: 856-864, 2000.
17. $\mathrm{Oh} \mathrm{H}$ and Schneider MD: The emerging role of telomerase in cardiac muscle cell growth and survival. J Mol Cell Cardiol 34: 717-724, 2002.

18. Li D and Rozen R: Maternal folate deficiency affects proliferation, but not apoptosis, in embryonic mouse heart. J Nutr 136: 1774-1778, 2006.

19. Chen JZ, Zhu JH, Wang XX, et al: Effects of homocysteine on number and activity of endothelial progenitor cells from peripheral blood. J Mol Cell Cardiol 36: 233-239, 2004.

20. Buemi M, Marino D, Di Pasquale G, et al: Effects of homocysteine on proliferation, necrosis, and apoptosis of vascular smooth muscle cells in culture and influence of folic acid. Thromb Res 104: 207-213, 2001.

21. Li D, Pickell L, Liu Y, Wu Q, Cohn JS and Rozen R: Maternal methylenetetrahydrofolate reductase deficiency and low dietary folate lead to adverse reproductive outcomes and congenital heart defects in mice. Am J Clin Nutr 82: 188-195, 2005.

22. Kwon C, Arnold J, Hsiao EC, Taketo MM, Conklin BR and Srivastava D: Canonical Wnt signaling is a positive regulator of mammalian cardiac progenitors. Proc Natl Acad Sci USA 104: 10894-10899, 2007. 